\title{
Corrigendum to "Time-Shift Correlation Algorithm for P300 Event Related Potential Brain-Computer Interface Implementation"
}

\author{
Ju-Chi Liu, ${ }^{1,2}$ Hung-Chyun Chou, ${ }^{3}$ Chien-Hsiu Chen, ${ }^{3}$ \\ Yi-Tseng Lin, ${ }^{4}$ and Chung-Hsien Kuo ${ }^{3,5}$ \\ ${ }^{1}$ Department of Internal Medicine, School of Medicine, College of Medicine, Taipei Medical University, Taipei 110, Taiwan \\ ${ }^{2}$ Division of Cardiology, Department of Internal Medicine, Shuang Ho Hospital, New Taipei City 235, Taiwan \\ ${ }^{3}$ Department of Electrical Engineering, National Taiwan University of Science and Technology, Taipei 106, Taiwan \\ ${ }^{4}$ Graduate Institute of Biomedical Engineering, National Taiwan University of Science and Technology, Taipei 106, Taiwan \\ ${ }^{5}$ Department of Biomedical Engineering, National Defense Medical Center, Taipei 114, Taiwan
}

Correspondence should be addressed to Chung-Hsien Kuo; chkuo@mail.ntust.edu.tw

Received 9 February 2017; Accepted 13 February 2017; Published 5 April 2017

Copyright (c) $2017 \mathrm{Ju}$-Chi Liu et al. This is an open access article distributed under the Creative Commons Attribution License, which permits unrestricted use, distribution, and reproduction in any medium, provided the original work is properly cited.

In the article titled "Time-Shift Correlation Algorithm for P300 Event Related Potential Brain-Computer Interface Implementation" [1], an acknowledgment should be added as follows:

The authors would like to thank the Ministry of Science and Technology (under Grant MOST 104-2221-E-011-140) and the Taipei Medical University/National Taiwan University of Science and Technology cross-university collaboration project (under Grant TMU-NTUST-103-07) for partial support of this work.

\section{References}

[1] J.-C. Liu, H.-C. Chou, C.-H. Chen, Y.-T. Lin, and C.-H. Kuo, "Time-shift correlation algorithm for P300 event related potential brain-computer interface implementation," Computational Intelligence and Neuroscience, vol. 2016, Article ID 3039454, 22 pages, 2016. 

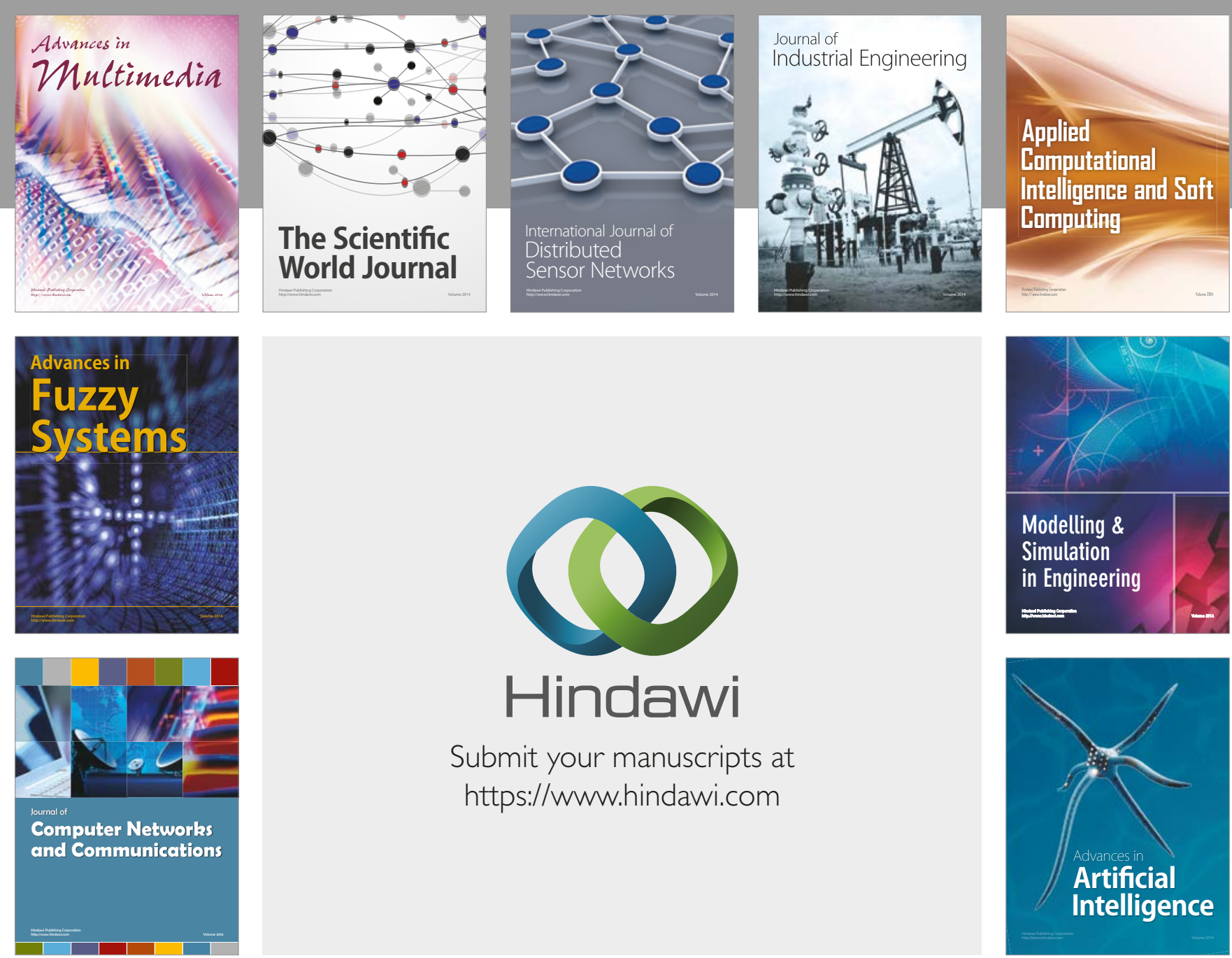

\section{Hindawi}

Submit your manuscripts at

https://www.hindawi.com
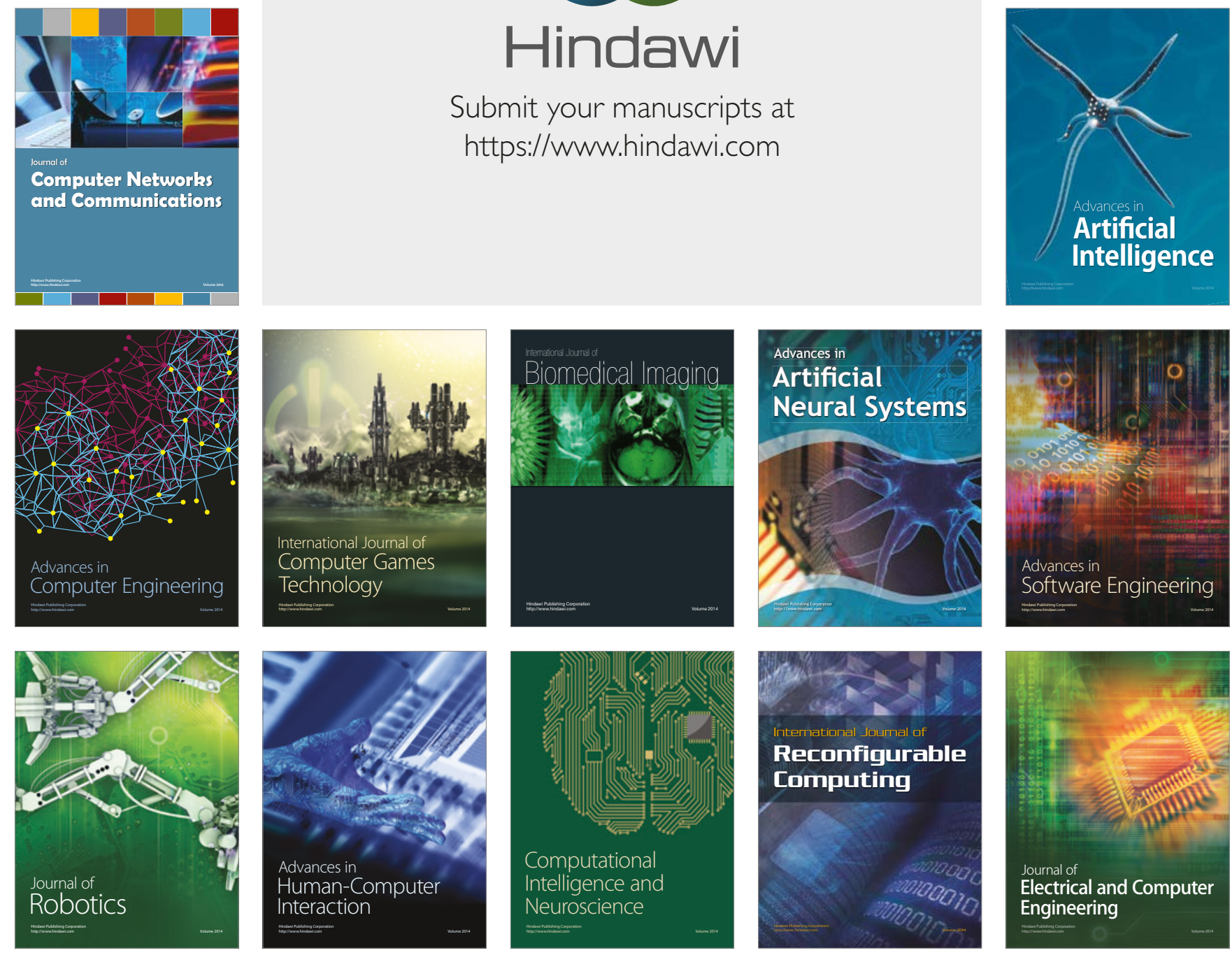